\title{
Pressure-induced variations of MLCT and ligand-centered luminescence spectra in square- planar platinum(II) complexes
}

\author{
Alexandre Rodrigue-Witchel, ${ }^{a}$ David L. Rochester, ${ }^{b}$ Shu-Bin Zhao, ${ }^{c}$ Kevin B. Lavelle, ${ }^{\mathrm{d}} \mathrm{J}$. A. \\ Gareth Williams, ${ }^{{ }^{b}}$ Suning Wang ${ }^{{ }^{*} \mathrm{c}}$, William B. Connick, ${ }^{{ }_{\mathrm{d}}}$ Christian Reber $^{{ }^{* a}}$ \\ ${ }^{\text {a }}$ Département de chimie, Université de Montréal, Montréal (Québec) H3C 3J7, Canada \\ ${ }^{\mathrm{b}}$ Department of Chemistry, Durham University, Durham DH1 3LE, U.K. \\ 'Department of Chemistry, Queen's University, Kingston, Ontario, K7L 3N6, Canada \\ ${ }^{\mathrm{d}}$ Department of Chemistry, University of Cincinnati, P.O. Box 210172, Cincinnati, Ohio 45221- \\ 0172, United States
}

\section{Keywords:}

Luminescence spectroscopy; variable-temperature spectroscopy; variable-pressure spectroscopy; platinum(II) complexes 


\begin{abstract}
Solid-state luminescence spectra of five crystalline platinum(II) complexes at variable pressure and temperature are presented and compared. Maxima occur between $14000 \mathrm{~cm}^{-1}$ and $15600 \mathrm{~cm}^{-1}$ (approximately $700 \mathrm{~nm}$ to $640 \mathrm{~nm}$ ) at ambient temperature and pressure. Spectra can be broad bands, characteristic of MLCT transitions, or vibronically structured, characteristic of intraligand transitions. Both pressure and temperature variations can lead to distinctive changes in the luminescence spectra that differ between these two types of transitions. MLCT band maxima show shifts on the order of $-20 \mathrm{~cm}^{-1} / \mathrm{kbar}$ to lower energy; in contrast, ligand-centered luminescence bands do not show significant shifts between ambient pressure and $40 \mathrm{kbar}$.
\end{abstract}




\section{Introduction}

Square-planar complexes with the $\mathrm{d}^{8}$ electron configuration are attractive building blocks for a variety of luminescent materials, due to their unsaturated coordination sphere.[1] Such compounds are studied as emitters for OLEDs[2] and for bioimaging [3], as they often show intense luminescence at room temperature.[1, 3] Aggregation is a key consideration because of its effects on luminescence energies and intensities.[4] Chemical variations can be used to modify the effects of aggregation.[5] Variations of ligands can lead to changes of both the molecular electronic structure[6] and the packing, making it challenging to explore individual contributing factors. Changes of the environment only, e.g. from crystals to solutions, or vapochromic effects, offer a more continuous variation. In this paper, we present results based on a continuous variation of the environment through external pressure leading to significant and characteristic effects on the luminescence spectra.[7]

Pressure-induced trends reveal particularly rich information, with shifts of luminescence maxima that can vary between $-400 \mathrm{~cm}^{-1} / \mathrm{kbar}[8]$ and $+50 \mathrm{~cm}^{-1} / \mathrm{kbar}$, a range wide enough to allow a classification of transitions and properties. Shifts for $\mathrm{d}-\mathrm{d}$ luminescence maxima are well documented and range from approximately $+10 \mathrm{~cm}^{-1} / \mathrm{kbar}$ to $+50 \mathrm{~cm}^{-1} / \mathrm{kbar}$. [9, 10] Shifts to lower energy, between $-100 \mathrm{~cm}^{-1} / \mathrm{kbar}$ and $-400 \mathrm{~cm}^{-1} / \mathrm{kbar}$, occur for compounds with strong metal-metal interactions such as tetracyanoplatinates. $[8,11]$ The results presented here fill the gap in these ranges and document the behavior of MLCT and ligand-centered transitions. 
The schematic structures and abbreviations for the five platinum(II) complexes used in this study are shown in Scheme 1. The complexes are neutral and have different ligands with extended $\pi$ systems, influencing stacking in the crystals and excited-state energies.

\section{Experimental}

The syntheses and characterization of all compounds have been published.[5, 6, 12-14] Luminescence and Raman spectra were measured using an InVia spectrometer coupled to an imagingmicroscope (Leica) and argon ion lasers. The excitation wavelength used was $488 \mathrm{~nm}$ forall luminescence measurements and $785 \mathrm{~nm}$ for the Raman spectra. Variable-temperature spectra were measured using a Linkam gas-flow microcryostat system and variable-pressure spectra with a diamond anvil cell (High Pressure Diamond Optics, Inc.). Pressure was determined by monitoring the luminescence of a ruby chip included with the sample in the diamond anvil cell. $[7,9]$ Ruby luminescence is denoted by asterisks in Figs 1 to 3.

\section{Spectroscopic Results}

Luminescence spectra of the five crystalline platinum(II) complexes are shown in Figs 1 to 5 at variable temperature and pressure. Values of the pressure-induced shifts are given in Table 1. BrNCNPtCl in Fig. 1 shows a single broad band at all temperatures and pressures explored. The band maximum shifts to lower energy with increasing pressure and decreasing temperature, as shown in Fig. 1. Trends of band maxima in the same direction at high pressure and low 
temperature are expected, as both variations lead to a decrease of the sample volume.[10] The maximum shifts by $+2.6 \mathrm{~cm}^{-1} / \mathrm{K}$. The band width increases from $1100 \mathrm{~cm}^{-1}$ to $1500 \mathrm{~cm}^{-1}$ between $80 \mathrm{~K}$ and room temperature, the characteristic signature of a vibronic intensity mechanism, as intensity increases mainly to the high-energy side of the electronic origin. This effect contributes to the shift of the maximum to higher energy as temperature increases.[15]

The luminescence spectra of PyNCNPtCl at variable pressure are presented in Fig. 2 and show a behavior similar to $\mathrm{BrNCNPtCl}$ in Fig. 1. A broad band with its maximum at approximately $14000 \mathrm{~cm}^{-1}$ again shifts to lower energy as pressure increases. Spectra recorded at variable temperature show two luminescence features at temperatures below $150 \mathrm{~K}$ : a broad band similar to Fig. $1 \mathrm{~b}$ with its maximum at approximately $14000 \mathrm{~cm}^{-1}$, and a more highly structured band at approximately $18000 \mathrm{~cm}^{-1}$. At room temperature, this second band disappears.

The NCNPtCl complex shows a broad band without resolved structure at pressures higher than 10 kbar, illustrated in Fig. 3a. At ambient pressure and variable temperature, the spectrum in Fig. $3 \mathrm{~b}$ shows some resolved structure, indicating a transition of a different nature than for the previous two complexes.

Fig. 4 shows luminescence spectra of the platinum(II) pincer complex Pt(POCN)Br.[13] A broad band with a maximum at approximately $15000 \mathrm{~cm}^{-1}$ is observed at room temperature and ambient pressure. The variable-pressure spectra in Fig. 4a show very little variation as pressure increases. The luminescence intensity increases at low temperature, as shown in Fig. 4b.

Fig. 5 shows luminescence spectra of $\operatorname{Pt}(\mathrm{BAM}) \mathrm{Me}_{2},[14]$ a complex with a dangling ligand methyl group situated perpendicular to the coordination plane containing the platinum(II) ion. 
Again, very small variations of the luminescence spectrum occur at variable pressure. The lowtemperature spectrum shows resolved vibronic structure with an average spacing similar in magnitude to the spectra in Fig. $3 b$.

Fig. 6 summarizes shifts of luminescence maxima with pressure. The slopes of the least-squares fits for all compounds are given in Table 1 . The first three complexes show a shift to lower energy on the order of $-15 \mathrm{~cm}^{-1} / \mathrm{kbar}$ to $-25 \mathrm{~cm}^{-1} / \mathrm{kbar}$, as shown in Figs 1 to 3 . It is interesting to note that $\mathrm{NCNPtCl}$ shows the smallest absolute value of these first three complexes, possibly due to the change of emitting state character as pressure increases: for the structured band observed at ambient pressure, no shift is expected, similar to the following two complexes. The spectra in Figs 4 and 5 do not show significant shifts of the maxima with pressure. The comparison of trends in Fig. 6 documents a surprising variation of shifts, despite the relatively similar energies of the band maxima for all compounds at ambient temperature and pressure, as given in Table 1. This diversity is in qualitative contrast to d-d luminescence from square-planar platinum(II) complexes where a shift to higher energy on the order of $+10 \mathrm{~cm}^{-1} / \mathrm{kbar}$ to $+30 \mathrm{~cm}^{-1} / \mathrm{kbar}$ is observed for many complexes[10], and, in exceptional cases, a strong shift to lower energy, on the order of $-100 \mathrm{~cm}^{-1} / \mathrm{kbar}$.[16] The behavior reported here for luminescence transitions of a different type falls in between these two limits, as discussed in the following. 


\section{Discussion}

The luminescence spectra in Figs 1 to 5 show a variety of different trends at variable pressure and temperature, indicative of different natures of the electronic transitions. The broad, featureless bands observed for $\mathrm{BrNCNPtCl}$ and $\mathrm{PyNCNPtCl}$ are typical for metal-to-ligand charge-transfer transitions (MLCT). In contrast, the bands with vibronic structure as observed for $\mathrm{NCNPtCl}$ and $\mathrm{Pt}(\mathrm{BAM}) \mathrm{Me}_{2}$ are typical of ligand-centered transitions, with the vibronic interval corresponding to a combination of ligand-centered stretching modes. The variations of the band maxima at variable pressure can be used to further probe these assignments.

Qualitative arguments correctly predict the pressure-induced variations of $\mathrm{d}$-d luminescence maxima observed for square-planar platinum(II) complexes: increasing pressure leads to slightly shorter metal-ligand bonds and a significant destabilization of the $\sigma$-antibonding LUMO level. The HOMO level can be nonbonding, $\pi$-bonding or $\pi$-antibonding, but its pressure-induced energy variation is smaller than for the LUMO, leading in all cases to a net increase of the HOMO-LUMO energy difference and the luminescence band maximum. The compounds here show a variation in the opposite direction, making a d-d assignment of the transition unlikely. The emitting state of an MCLT transition involves a ligand-centered LUMO, and pressure is not expected to significantly change the energy of this excited state, as length changes of strong covalent ligand bonds are much smaller than those of weaker metal-ligand bonds. In a qualitative picture, the LUMO energy remains therefore unchanged. The metal-centered HOMO level has $\pi$ antibonding character, in particular towards the halide ligand most likely pushed closer to the platinum(II) center by an external pressure, and its energy is expected to increase with pressure. 
These qualitative trends point toward a decrease of the transition energy, as observed by the shift of luminescence maxima to lower energies with increasing pressure for the complexes in Figs 1 to 3. BrNCNPtCl and PyNCNPtCl illustrate this expected trend to lower energy, as shown in Figs 1a, 2a and 6. NCNPtCl in Fig. $3 \mathrm{a}$ is an interesting case: as pressure increases, the band with resolved vibronic structure disappears and a broad band dominates, with a maximum that shifts to lower energy as pressure increases, somewhat lower in magnitude than the shifts for BrNCNPtCl and PyNCNPtCl. The change of the band shape can again be qualitatively rationalized in the same way: at ambient pressure, a ligand-centered band is observed, but the destabilization of metal-centered orbitals as metal-ligand bonds are compressed leads to a change of HOMO to a metal-centered level at higher pressure. Metal-metal interactions in platinum(II) compounds have been shown to lead to shifts to lower energy larger by an order of magnitude than the effects reported here. In all these systems, distances lower than $3.5 \AA$ perpendicular to the coordination plane are observedbetween the platinum(II) center and neighboring metal or ligand atoms. Metalmetal distances for $\mathrm{NCNPtCl}$ and $\mathrm{Pt}(\mathrm{POCN}) \mathrm{Br}$ are $4.8 \AA[12]$ and $8.8 \AA$,[13] well above the threshold for efficient metal-metal interactions, leading to low absolute values of pressureinduced shifts of luminescence maxima, as given in Table 1.BrNCNPtCl and PyNCNPtCl show more pronounced shifts to low energy with pressure, possibly due to a contribution from intermolecular interactions involving electron density of the platinum(II) center perpendicular to the coordination plane and ligand fragments of a neighboring complex, destabilizing the HOMO orbital. In comparison, classic complexes such as $\mathrm{Pt}\left(2,2^{\prime}\right.$-bipyridine $) \mathrm{Cl}_{2}$ with $\mathrm{MLCT}$ luminescence show even stronger shifts to low energy due to metal-metal interactions and variations in tendencies due to structural phase transitions.[17] The observed shifts of the 
luminescence maxima to lower energy are therefore most likely caused by a combination of intermolecular and intramolecular effects and define the typical range for MLCT transitions.

The predominantly ligand-centered transitions in Figs 4 and 5 do not show an appreciable shift of their band maxima, as expected for the very small structure changes, leaving HOMO and LUMO energies unchanged and leading to the zero shifts reported in Table 1.

The variations of the luminescence spectra with temperature reveal that transitions of different nature are close in energy for the five compounds. $\mathrm{BrNCNPtCl}$ shows a shift of the maximum to higher energy as temperature increases, but no evidence of a different transition is observed in the spectra recorded at variable temperature in Fig. 1. The shift with temperature is due to both vibronic intensity gains,[15] leading strictly to a broadening on the high-energy side of the band, and intermolecular interactions, qualitatively leading to changes of the electronic origin and on the low-energy side of the band and increasing the total shift of $\mathrm{E}_{\max }$ to higher energy as temperature increases, indirect evidence for the influence of intermolecular interactions.

The variable-temperature luminescence spectra for $\mathrm{PyNCNPtCl}$ in Fig. $2 \mathrm{~b}$ show a change of the type of transition: at low temperature, a structured band appears on the high-energy side of the broad band, indicative of ligand-centered transitions. One likely physical origin of this spectrum are slightly different complexes with different luminescence transitions. At high temperature a broad band without any structure dominates, possibly due to efficient energy transfer deactivating the luminophores with the higher-energy luminescence bands and showing the typical shift to lower energy expected for an MLCT band. Variable-temperature Raman spectra do not show any 
changes indicative of a phase transition that could lead to the change observed in the luminescence spectra.

The variable-temperature luminescence spectra of $\mathrm{NCNPtCl}$ in Fig. $3 \mathrm{~b}$ show the typical behavior of a ligand-centered transition with resolved vibronic structure at low temperature and loss of resolution as temperature increases. For the complexes in Figures 4 and 5, the band maxima do not show significant shifts with temperature.

The vibronic structure for $\mathrm{NCNPtCl}$ and $\mathrm{Pt}(\mathrm{BAM}) \mathrm{Me}_{2}$ can be quantitatively analyzed using the time-dependent theory of electronic spectroscopy.[18-20]Harmonic potential energy surfaces defined by vibrational frequencies identical for the ground and emitting states are assumed. Initial values for the frequencies, the energy of the electronic origin $E_{00}$ and the width $\Gamma$ of each vibronic transition were estimated from the experimental spectra and adjusted to improve agreement between calculated and experimental spectra. The offsets $\Delta$ between ground and emitting state potential energy minima along normal coordinates were also adjusted. Calculated spectra are in good agreement with experimental data, as shown in Fig. 7, and all parameter values used for the calculations are given in Table 2. The shape and overall width of the spectra are defined by the intensity distribution within the vibronic progressions, arising from small but significant offsets of the emitting state potential energy surface along ligand-centered normal coordinates, most likely stretching modes given their frequencies on the order of $1300 \mathrm{~cm}^{-1}$ to $1500 \mathrm{~cm}^{-1}$. Such modes are not expected to be affected by a small perturbation such as external pressure, confirmed by the spectra in Figs 4 and 5 which do not show any significant variations of the vibronic intervals over the pressure range explored here. The resolved vibronic structure of 
the calculated spectra is easily removed by increasing the value of $\Gamma$ defining the width of each vibronic transition. Such unresolved spectra are useful to determine the values of luminescence band maxima, leading to the $\mathrm{E}_{\max }$ values denoted by open symbols in Fig. 6 .

\section{Summary}

The comparison of luminescence spectra of five platinum(II) complexes shows that transitions with predominant MLCT character show variable-pressure shifts to lower energy, likely due to a destabilization of the metal-centered HOMO level with the ligand-centered LUMO energy not varying significantly. For ligand-centered transitions, neither HOMO nor LUMO energies change significantly, leading to band maxima that do not show any shift. These results add to the rich variety of luminescence properties for square-planar $\mathrm{d}^{8}$ complexes, even in the absence of metalmetal interactions. The influence of other neighboring groups on MLCT and ligand centered transitions will be explored and quantitatively characterized.

\section{Acknowledgements}

Financial support from the Natural Sciences and Engineering Research Council (Canada) is gratefully acknowledged. CR acknowledges travel funding from the Hans and Marlies Zimmer

International Scholar Fund, University of Cincinnati. DLR thanks EPSRC and Durham University for a studentship. 


\section{References}

[1] V.W.-W. Yam, V.K.-M. Au, S.Y.-L. Leung, Chem. Rev., 115 (2015) 7589.

[2] M. Cocchi, J. Kalinowski, V. Fattori, J.A.G. Williams, L. Murphy, Appl. Phys. Lett., 94 (2009) 073309.

[3] J.A.G. Williams, Chem. Soc. Rev., 38 (2009) 1783. L. Murphy and J. A. G. Williams, Top. Organomet. Chem. 28 (2010) 75. E. Baggaley, S. W. Botchway, J. W. Haycock, H. Morris, I. V. Sazanovich, J. A. G. Williams, J. A. Weinstein, Chem. Sci., 5 (2014) 879.

[4] D.R. McMillin, J.J. Moore, Coord. Chem. Rev., 229 (2002) 113. E. Rossi, L. Murphy, P. L. Brothwood, A. Colombo, C. Dragonetti, D. Roberto, R. Ugo, M. Cocchi, J. A. G. Williams, J. Mater. Chem. 21 (2011) 15501.

[5] S.J. Farley, D.L. Rochester, A.L. Thompson, J.A.K. Howard, J.A.G. Williams, Inorg. Chem., 44 (2005) 9690.

[6] D.L. Rochester, S. Develay, S. Zális, J.A.G. Williams, Dalton Trans., (2009) 1728.

[7] K.L. Bray, Top. Curr. Chem., 213 (2001) 1.

[8] E. Leyrer, F. Zimmermann, J.I. Zink, G. Gliemann, Inorg. Chem., 24 (1985) 102.

[9] C. Reber, J.K. Grey, E. Lanthier, K.A. Frantzen, in: J.P. Fackler, L.R. Falvello (Eds.) Techniques in Inorganic Chemistry, CRC Press, Boca Raton, 2010, pp. 181.

[10] S. Poirier, R.J. Robert, D. Le, D.B. Leznoff, C. Reber, Inorg. Chem., 54 (2015) 3728. 
[11] G. Gliemann, H. Yersin, Struct. Bond., 62 (1985) 87.

[12] D.J. Cárdenas, A.M. Echavarren, M.C. Ramírerz de Arellano, Organometallics, 18 (1999) 3337.

[13] K.B. Lavelle, L. Gutierrez, J.A. Krause, W.B. Connick, J. Organomet. Chem., 785 (2015) 1.

[14] S.-B. Zhao, G.-h. Liu, D. Song, S. Wang, Dalton Trans., (2008) 6953.

[15] S. Poirier, P. Guionneau, D. Luneau, C. Reber, Can. J. Chem., 92 (2014) 958.

[16] G. Levasseur-Thériault, C. Reber, C. Aronica, D. Luneau, Inorg. Chem., 45 (2006) 2379.

[17] O.S. Wenger, S. García-Revilla, H.U. Güdel, H.B. Gray, R. Valiente, Chem. Phys. Lett., 384 (2004) 190.

[18] E.J. Heller, Acc. Chem. Res., 14 (1981) 368-375.

[19] J.I. Zink, K.-S.K. Shin, Adv. Photochem., 16 (1991) 119.

[20] J.I. Zink, Coord. Chem. Rev., 211 (2001) 69. 


\section{Table 1}

Comparison of emission maxima and shifts with pressure for all five platinum(II) complexes.

\begin{tabular}{|l|l|l|}
\hline Compound & $\begin{array}{l}\mathrm{E}_{\max }\left(\mathrm{cm}^{-1}\right) \\
\text { ambient } \mathrm{p} \text { and } \mathrm{T}\end{array}$ & $\begin{array}{l}\text { Shift at variable } \mathrm{p} \\
\left(\mathrm{cm}^{-1} / \mathrm{kbar}\right)\end{array}$ \\
\hline $\mathrm{NCNPtCl}$ & 14600 & -15 \\
\hline PyNCNPtCl & 14300 & -25 \\
\hline BrNCNPtCl & 14000 & -24 \\
\hline $\mathrm{Pt}(\mathrm{POCN}) \mathrm{Br}$ & 15200 & 0 \\
\hline $\mathrm{Pt}(\mathrm{BAM}) \mathrm{Me}_{2}$ & 15600 & 0 \\
\hline
\end{tabular}

\section{Table 2}

Fit parameters used to calculate the luminescence spectra shown in Fig. 7.

\begin{tabular}{|l|l|l|l|l|}
\hline Compound & $\begin{array}{l}\Gamma\left(\mathrm{cm}^{-1}\right) \\
\text { (width of } \\
\text { vibronic } \\
\text { transitions) }\end{array}$ & $\begin{array}{l}\mathrm{E}_{00}\left(\mathrm{~cm}^{-1}\right) \\
\text { (energy of } \\
\text { electronic } \\
\text { origin) }\end{array}$ & $\begin{array}{l}\text { hw }\left(\mathrm{cm}^{-1}\right) \\
\text { (vibrational } \\
\text { frequencies) }\end{array}$ & $\begin{array}{l}\Delta \text { (dim. less) } \\
\text { (offset of } \\
\text { potential energy } \\
\text { minima) }\end{array}$ \\
\hline $\mathrm{NCNPtCl}$ & 380 & 17000 & 1300 & 1.75 \\
\hline $\operatorname{Pt}(\mathrm{BAM}) \mathrm{Me}_{2}$ & 280 & 18170 & 1520,975 & $1.9,1.25$ \\
\hline
\end{tabular}




\section{Figure Captions}

Scheme 1. Schematic structures and abbreviations used for the title compounds. Top to bottom: PyNCNPtCl:1,3,5-tri(2-pyridyl)benzene platinum(II) chloride; $\mathrm{Pt}(\mathrm{BAM}) \mathrm{Me}_{2}$ : bis(7-azaindol-1yl)methanePt $\left(\mathrm{CH}_{3}\right)_{2}$; NCNPtCl: 1,3-di(2-pyridyl)benzene platinum(II) chloride; $\mathrm{Pt}(\mathrm{POCN}) \mathrm{Br}$ : $\mathrm{k}^{\mathrm{P}}, \mathrm{k}^{\mathrm{C}}, \mathrm{k}^{\mathrm{N}}-\left[2,6-\left(\mathrm{iPr}_{2} \mathrm{PO}\right)-\left(\mathrm{C}_{6} \mathrm{H}_{3}\right)\left(\mathrm{CH}_{2}\left[\mathrm{c}-\mathrm{N}\left(\mathrm{CH}_{2}\right)_{4} \mathrm{O}\right]\right)\right] \mathrm{PtBr} ; \quad$ BrNCNPtCl: $\quad$ 1-bromo-3,5-di(2 pyridyl)benzene platinum(II) chloride.

Fig. 1. Luminescence spectra of $\mathrm{BrNCNPtCl}$ at variable pressure (16 kbar, $28 \mathrm{kbar}$ and $40 \mathrm{kbar}$ ) (a) and at variable temperature $(80,130,180,230,270$ and $290 \mathrm{~K})$ (b). The peaks denoted by * are ruby luminescence used for pressure calibration.

Fig. 2. Luminescence spectra of PyNCNPtCl at variable pressure (a) and at variable temperature (b). The peaks denoted by * are ruby luminescence used for pressure calibration.

Fig. 3. Luminescence spectra of $\mathrm{NCNPtCl}$ at variable pressure (a) and at variable temperature (b). The peaks denoted by $*$ are ruby luminescence used for pressure calibration

Fig. 4. Luminescence spectra of $\mathrm{Pt}(\mathrm{POCN}) \mathrm{Br}$ at variable pressure (a) and at variable temperature (b).

Fig. 5. Luminescence spectra of $\mathrm{Pt}(\mathrm{BAM}) \mathrm{Me}_{2}$ at variable pressure (a) and at variable temperature (b).

Fig. 6. Luminescence band maxima at variable pressure. Top to bottom: open circles denote luminescence maxima for $\mathrm{Pt}(\mathrm{BAM}) \mathrm{Me}_{2}$; open squares $\mathrm{Pt}(\mathrm{POCN}) \mathrm{Br}$; solid circles: PyNCNPtCl, solid triangles: $\mathrm{BrNCNPtCl}$, solid squares $\mathrm{NCNPtCl}$. Least-square linear fits are shown for all five complexes. 
Fig. 7. Calculated (solid lines) and experimental (dotted lines) luminescence spectra for $\mathrm{NCNPtCl}$ at $298 \mathrm{~K}$ (a) and $\mathrm{Pt}(\mathrm{BAM}) \mathrm{Me}_{2}$ at $83 \mathrm{~K}$ (b). 
Figure 1

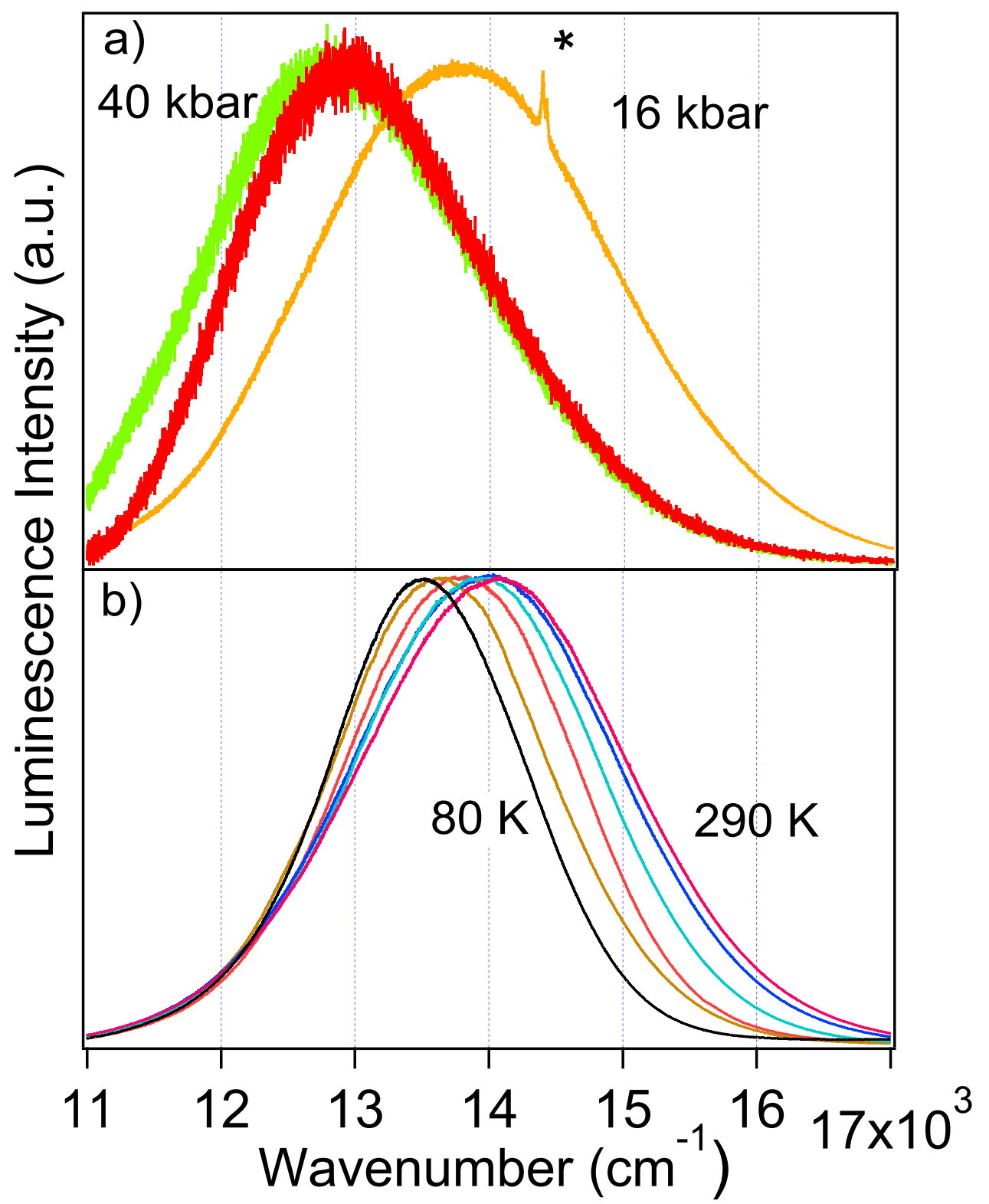


Figure 2

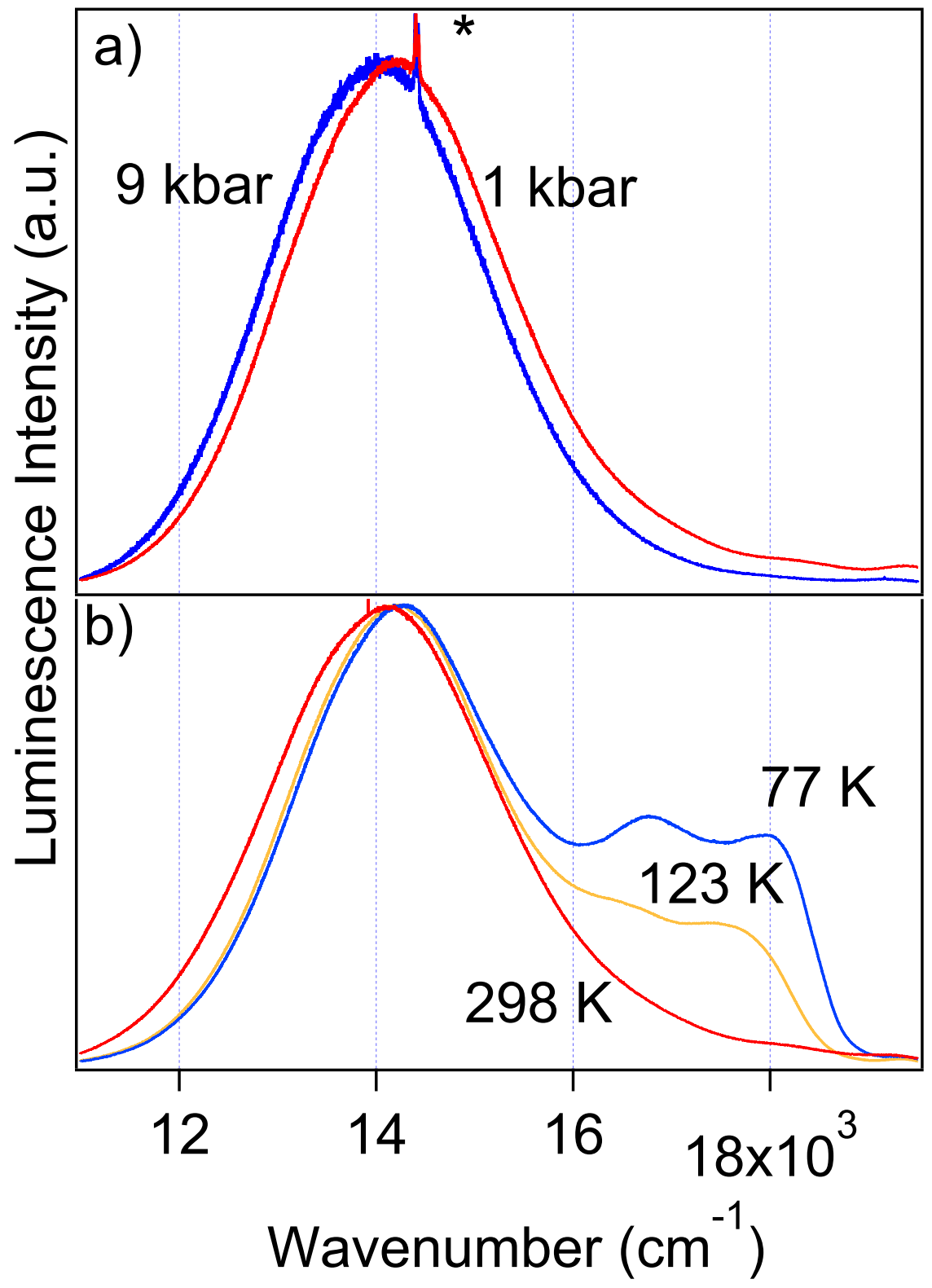


Figure 3

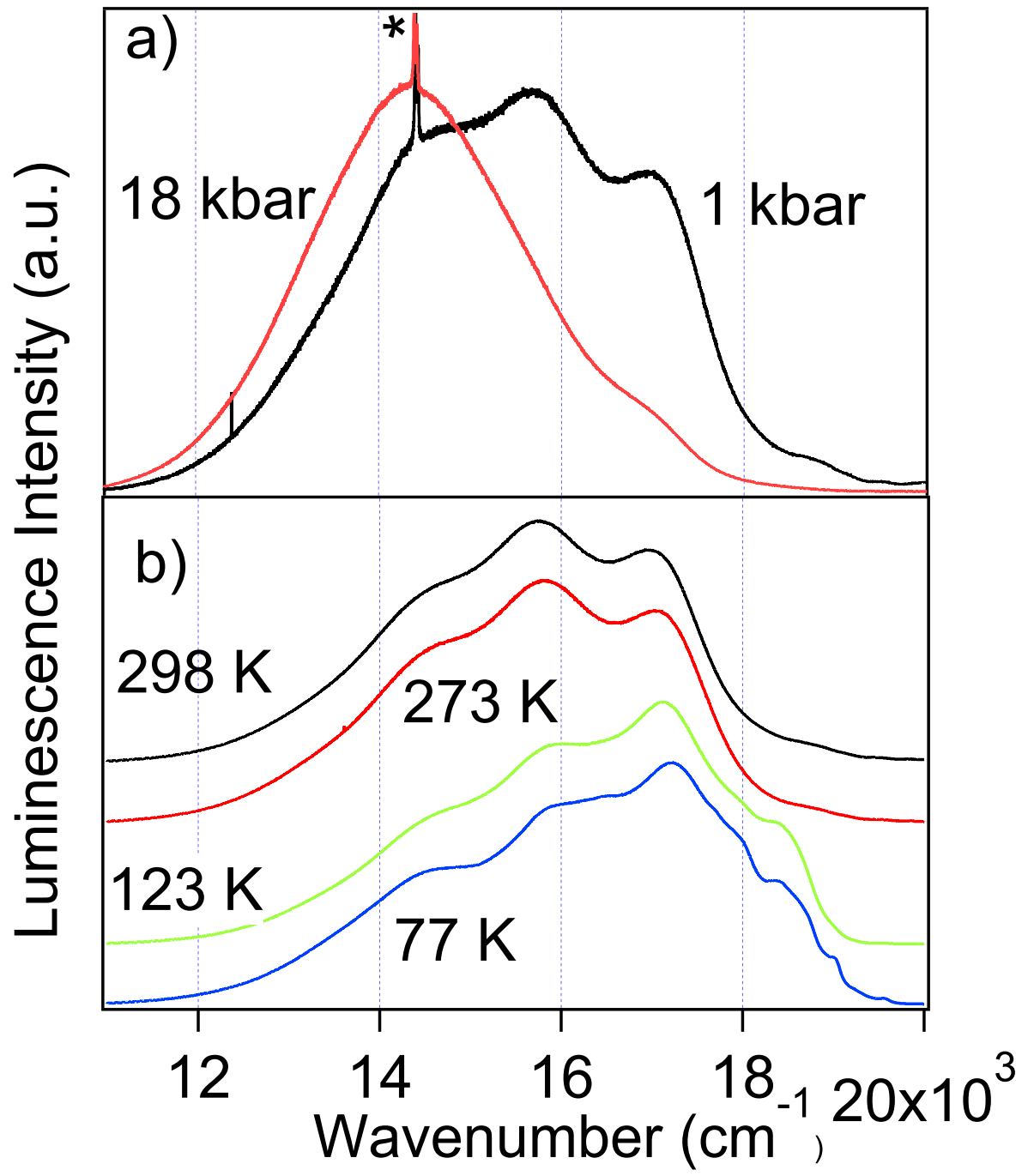


Figure 4

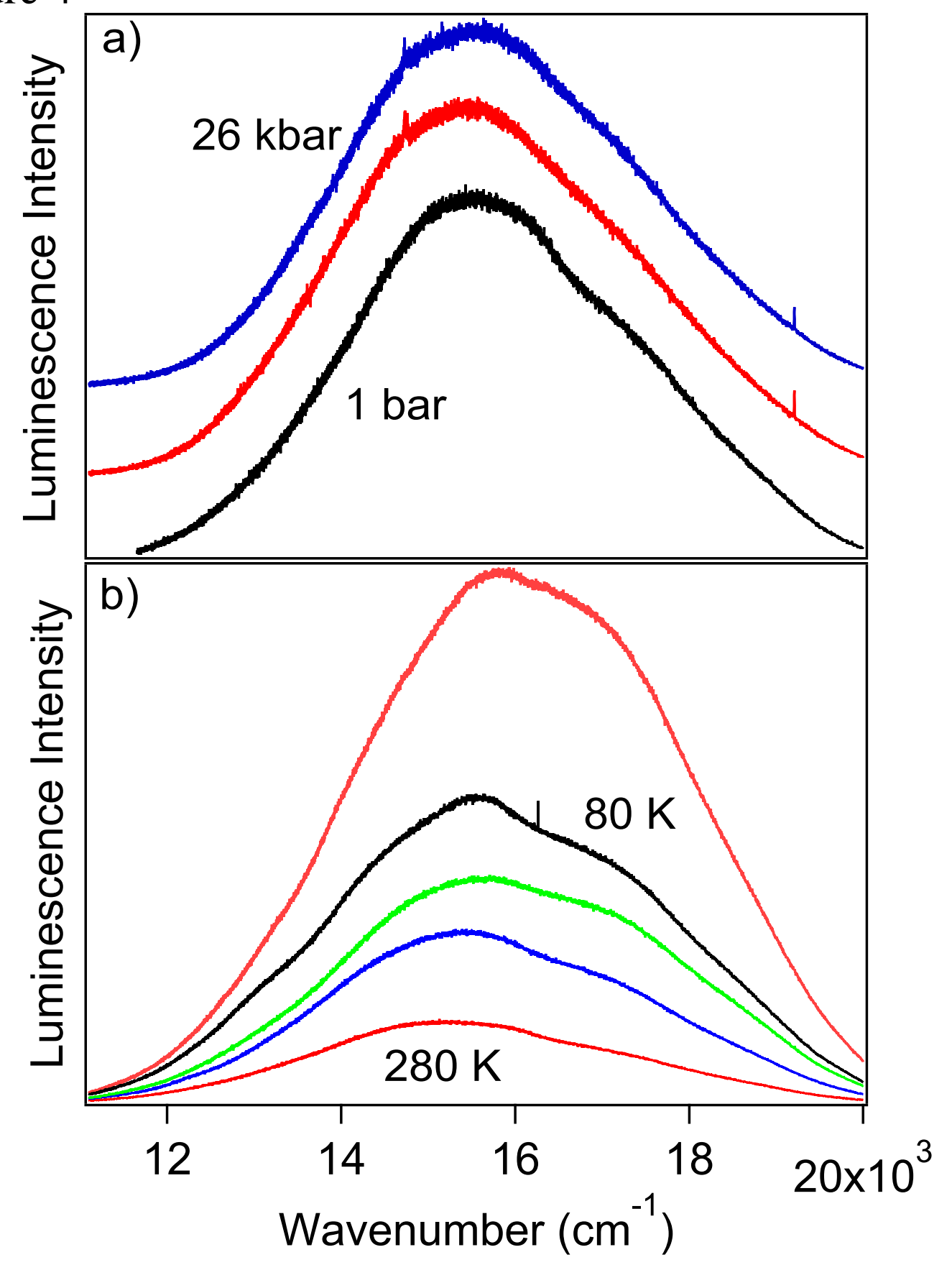


Figure 5
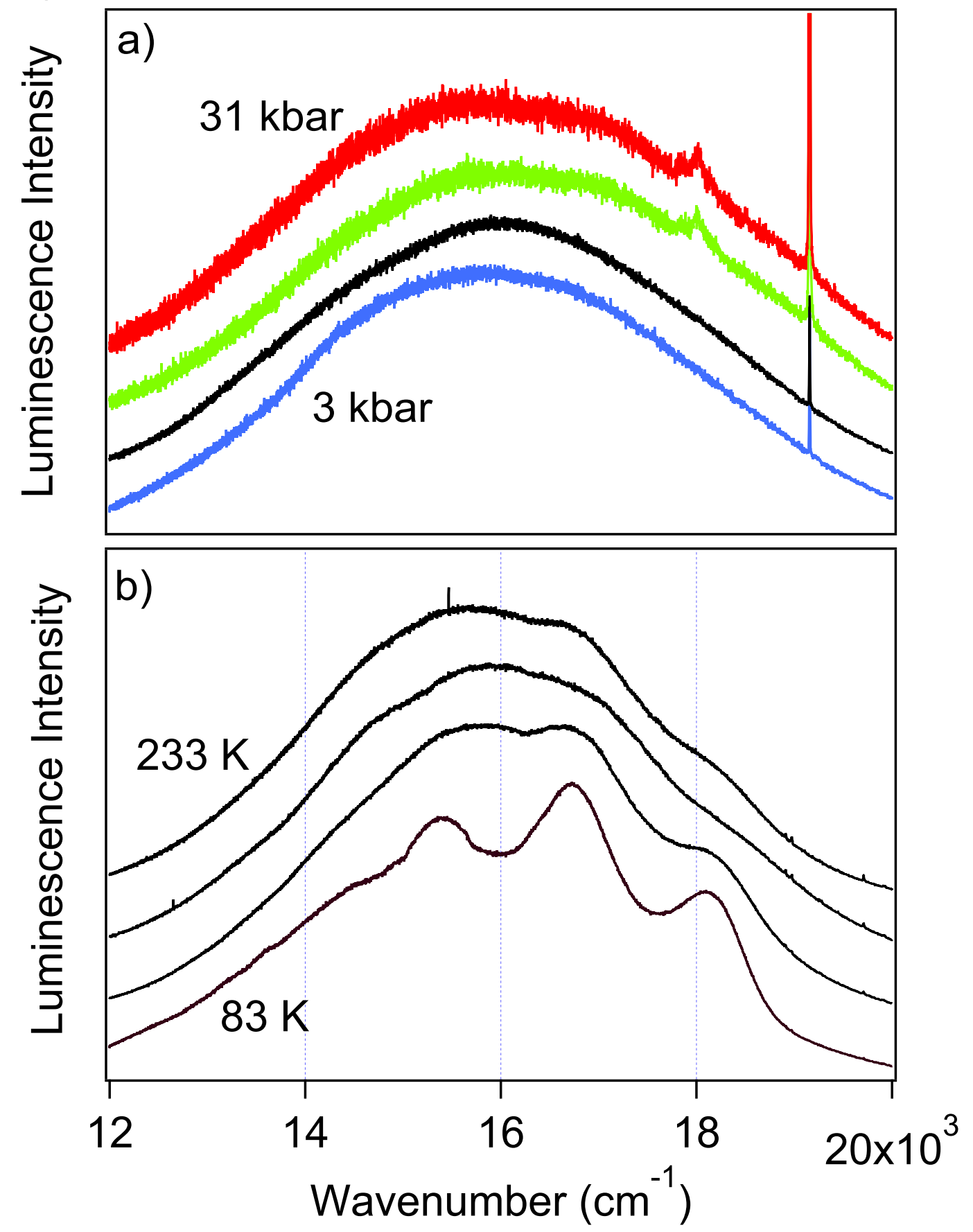
Figure 6

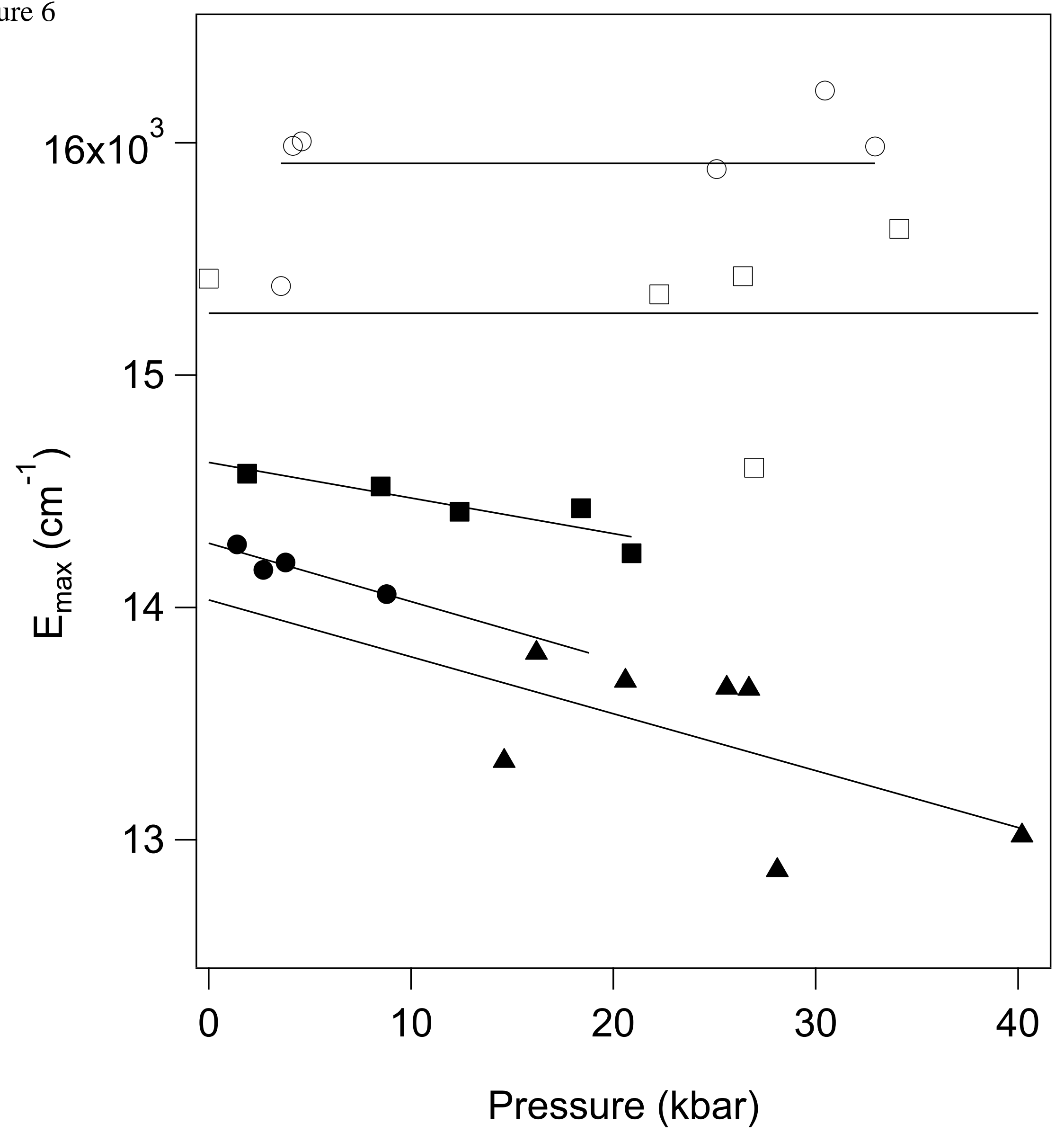


Figure 7

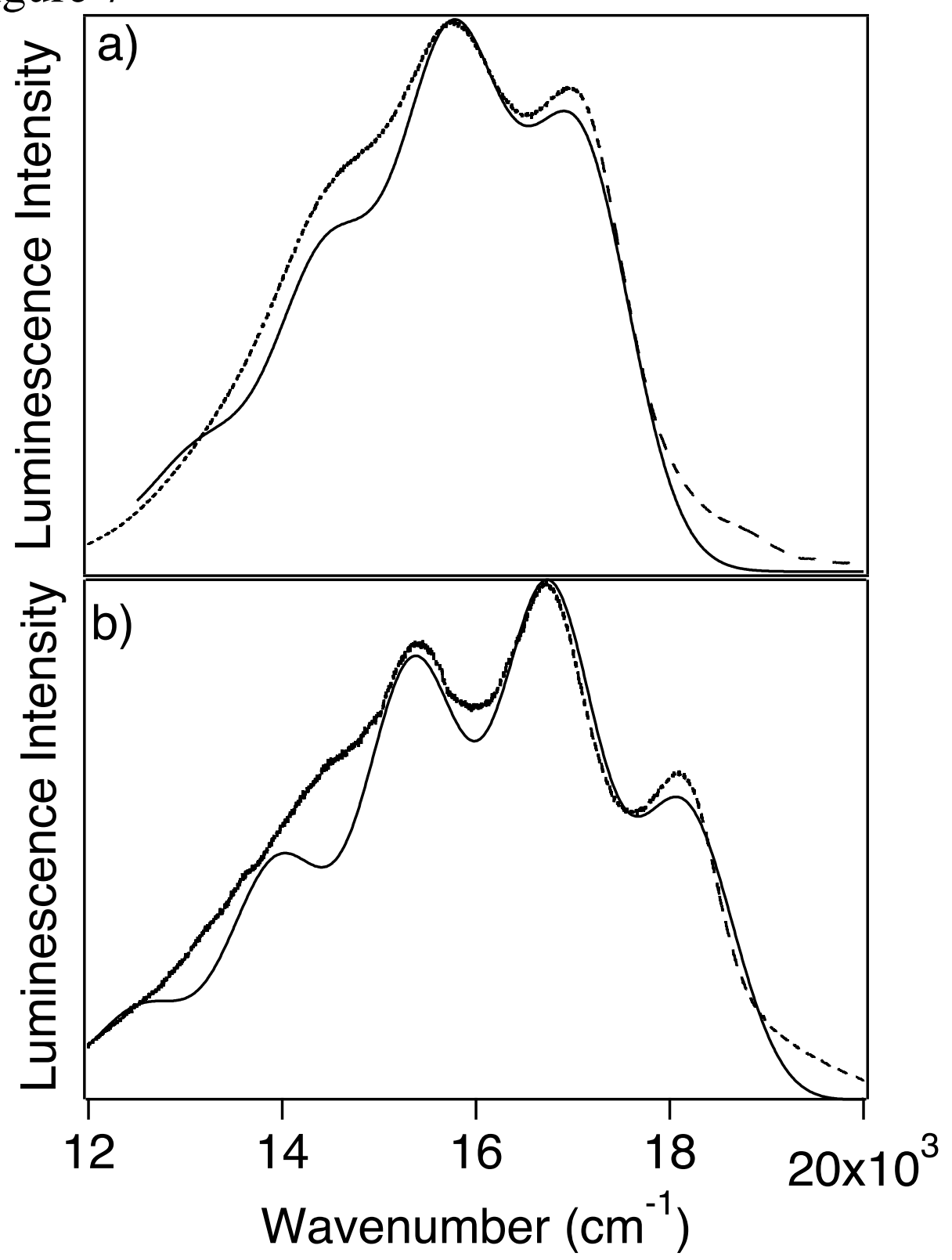




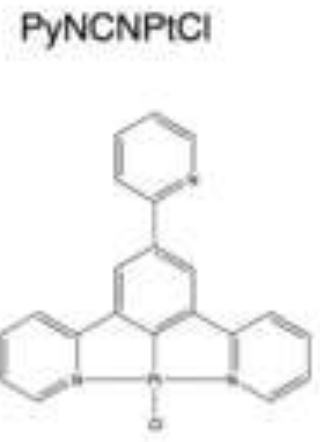

$$
\mathrm{P} t(\mathrm{BAM}) \mathrm{Me}_{2}
$$

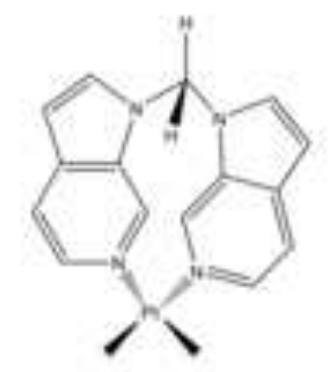

NCNPICI

$\mathrm{Pt}(\mathrm{POCN}) \mathrm{Br}$
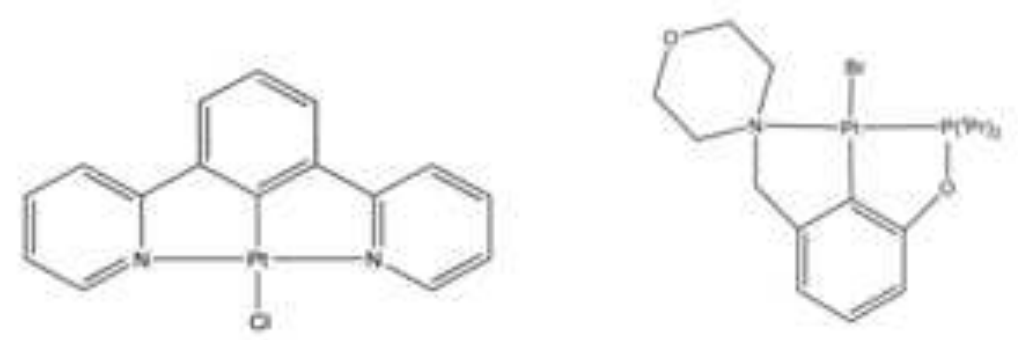

BrNCNPtCl

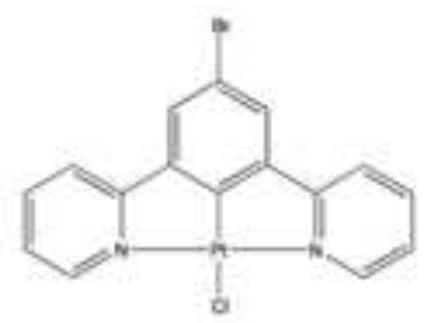

